Journal of Applied Analysis

Vol. 13, No. 2 (2007), pp. 235-247

\title{
WEINSTEIN'S TECHNIQUE FOR A CLASS OF PARABOLIC PROBLEMS
}

\author{
L. RAGOUB \\ Received September 28, 2005 and, in revised form, June 9, 2007
}

\begin{abstract}
We investigate a class of over-determined parabolic problems involving a non-constant boundary condition. The Weinstein's technique known for the elliptic problems is extended to the parabolic one by means of auxiliary functions and Green classical formula.
\end{abstract}

\section{INTRODUCTION}

Maximum principles are power tools for partial differential equations both of elliptic and parabolic equations [5], [9], [10], [11], in particular the study of the over-determined boundary value problems is of great interests. The two different classes elliptic and parabolic problems are investigated by many authors (see [6], [7], [15], [21]) where mostly in these kind of situations, the normal derivative of the solution already used has a constant value on the boundary $\partial \Omega$ of $\Omega$, where $\Omega$ is assumed to be bounded, and in some cases convex. We refer for existence and uniqueness of solutions of elliptic problems to [2], [3], [4] and the references therein. In 1971, Serrin [18]

2000 Mathematics Subject Classification. 28D10, 35B05, 35B50, 35J25, 35J60, 35J65.

Key words and phrases. Maximum principle, Weinstein's technique, initial parabolic problems.

This work has been funded by Research Center under Project No. (Math/2006/46).

ISSN 1425-6908（C) Heldermann Verlag. 
in his elegant paper considered the following problem, called Saint-Venant problem:

$$
\Delta u=-1 \text { in } \Omega, \quad u=0 \text { and } \frac{\partial u}{\partial n}=\text { const }
$$

on the boundary $\partial \Omega$. He showed that the only configuration of this domain is the ball. In his proof he used the moving plane method together with maximum principles of E. Hopf [9], [10], [19] while Weinberger [21] proved shortly the result by constructing a new auxiliary function. From these new techniques arising in a class of over-determined problems was derived many results. Unfortunately, for the elliptic boundary value problems we have limit success when the normal derivative is not constant on the boundary $\partial \Omega$. For example, in order to compare the solution $u$ with the reflected solution $u^{\lambda}$ some difficulties are met when the moving plane is involved. In this direction many efforts are done (see [1], [8], [13], [22]) and Weinstein's technique seems best way even if we have partial results. Basically, the idea of Weinstein is to reduce the original problem to a new one where the method of Serrin [18] is applicable. Alternatively, using the continuous Steiner symmetrization with domain derivative, F. Brock [1] has successfully proved that the domain is an $N$-ball when the normal derivative depends on the radius $r$ of the form $\psi(r)$. With a weaker condition than of F. Brock, Tewodros [20] used an other approach based on auxiliary functions and maximum principles where he treated the Saint-Venant problem over-determined by

$$
\frac{\partial u}{\partial n}=-c r,
$$

he showed too, that $\Omega$ is an $N$-ball. Now the concerns herein, is to extend the argument of Weinstein to a class of parabolic over-determined problems. The ingredients of this investigation are auxiliary functions and classical formula of Green. Although the proof made in this paper inspired from A. Weinstein, the difficulties for a class of parabolic problems are met again.

This paper is organized as follows. In Section 2, we consider an initial parabolic problem in $\mathbb{R}^{2}$, defined in an angular domain $\Omega$ where the normal derivative of $u$ takes two different variables data on the boundary $\partial \Omega$ and we show that the only configuration of the non-radial component $\Gamma$ is an arc of circle for two particular cases. In Section 3, we investigate an initial parabolic problem in $\mathbb{R}^{3}$ where the region considered $\Omega$ is tubular. We show that the solution is axially symmetric and the corresponding components of the domain are two co-axial circular cylinders.

In the sequel, for each section we assume that the solution for the problem in consideration exists. In addition the domain $\Omega$ will be supposed to be of class $C^{2}$ and its boundary $\partial \Omega$ of class $C^{2+\varepsilon}$. 


\section{ON A CLASS OF INITIAL PARABOLIC BOUNDARY VALUE PROBLEMS}

Let $\Omega$ be an angular domain defined in polar coordinates $(r, \theta)$ by

$$
\Omega:=\left\{(r \cos \theta, r \sin \theta) \in \mathbb{R}^{2} \mid 0<r<r(\theta), \theta \in\left[0, \frac{\pi}{\alpha}\right]\right\},
$$

where $(r, \theta)$ is a given positive function, bounded, defined on $[0, \pi / \alpha]$, and $\alpha \geq 1$. We consider the initial boundary value problem

$$
\begin{aligned}
\Delta u & =u_{t} & & \text { in } \Omega \times(0, \infty), \\
u(x, y, t) & =0 & & \text { on } \partial \Omega \times(0, \infty), \\
u(x, y, 0) & =\left(r^{\alpha} \sin \alpha \theta\right) g(r) & & \text { in } \Omega,
\end{aligned}
$$

where $g$ is supposed to be of class $C^{2}$ on $\bar{\Omega}$ and $r:=\sqrt{x^{2}+y^{2}}$.

In (1), $\Delta$ denotes the Laplace operator in $\mathbb{R}^{2}$, and $\partial \Omega$ is the boundary of $\Omega$ assumed sufficiently regular. We use the comma notation with the summation convention, i.e. the comma denotes partial differentiation and the repeated index indicates summation over $i=1,2$, as

$$
u_{, i i}=\frac{\partial^{2} u}{\partial x^{2}}+\frac{\partial^{2} u}{\partial y^{2}}=\Delta u, \quad u_{, i} u_{, i}=|\nabla u|^{2}=\left(\frac{\partial u}{\partial x}\right)^{2}+\left(\frac{\partial u}{\partial y}\right)^{2} .
$$

In the next statement we formulate our mean result

Theorem 2.1. We assume that $u$ satisfies the problem (1)-(3) in a bounded domain $\Omega$, regular and convex and its boundary $\partial \Omega$ of class $C^{2+\varepsilon}$. If we impose furthermore the following boundary condition

$$
\frac{\partial u}{\partial n}=-c(t) r^{\alpha} \sin \alpha \theta \text { on } \Gamma, \quad t>0,
$$

where $c(t)$ is a positive function in $t$, and $\Gamma$ denotes the non-radial component of the boundary $\partial \Omega$. Then the domain $\Omega$ is a circular sector of angle $\frac{\pi}{\alpha}$.

The aim of this theorem is to determine for which kind of $\Gamma$ the overdetermined problem (1)-(4) possesses a solution. The desired geometrical form is determined for some particular situations, this is due to the fact that when the original problem is not transformed to the classical problem of Serrin, we are unable to prove or disprove in $\mathbb{R}^{2}$ the claimed configuration stated in Theorem 2.1.

In this section, we conjecture that the only configuration of $\Gamma$ for which the over-determined problem (1)-(4) possesses a solution, is an arc of circle centered at the origin. We investigate this conjecture for two particular cases, $\alpha=1$ and $\alpha=2$ by means of Weinstein's technique, already used by Payne [12], Payne and Weinstein [13] for a class of elliptic problems. 
Since this method seems with limited success, the problem is still open for $\alpha \geq 3$. We begin by the first case, when $\alpha=1$. In this situation the over-determined condition (4) takes the form

$$
\frac{\partial u}{\partial n}=-c(t) y \text { on } \Gamma>0, \quad t>0 .
$$

The corresponding domain is situated in the first quadrant $y>0$. Upon, we prove that the geometrical form of the resulting over-determined problem $(1)-(3),(5)$ is a semi-disc centered at the origin. As a first step, we reduce the over-determined problem (1)-(4) to the simplest one in the sense that the normal derivative of the solution $u$ will be constant on the boundary $\partial \Omega$ after what Green classical formula leads to the desired result. We make a clear vision by considering the new reduced problem in more dimensional space.

We set

$$
u(x, y, t)=y v(x, y, t) .
$$

We see easily that the function $v$ defined in (6) satisfies the following conditions

$$
\begin{aligned}
\Delta v+2 \frac{v_{y}}{y} & =v_{t}, \quad v>0 \text { in } \Omega, & & t>0, \\
v(x, y, t) & =0 \text { on } \Gamma, & & t>0, \\
\frac{\partial v}{\partial n} & =-c(t) \text { on } \Gamma, & & t>0, \\
v(x, y, 0) & =g(r), & & (x, y) \in \Omega .
\end{aligned}
$$

For reason of compatibility, the function $g$ appearing in (10) must satisfy $g=0$ on the boundary $\Gamma$ of $\Omega$. Furthermore, we observe that on the $x$-axis the normal derivative of $v$ is trivial, this is due to the elementary computation

$$
\begin{aligned}
\frac{\partial v}{\partial y} & =\left(\frac{u}{y}\right)_{y} \\
& =\frac{y u_{y}-u}{y^{2}} .
\end{aligned}
$$

Employing (1) and (2), we get

$$
\left.\frac{\partial v}{\partial y}\right|_{y=0}=\lim _{y \rightarrow 0} \frac{u_{y y}}{2}=0 .
$$

Now in order to reduce the problem in higher dimension, we introduce a new function $\Phi$ defined by

$$
\Phi\left(x_{1}, x_{2}, x_{3}, x_{4}, t\right)=v(x, y, t), \quad(x, y) \in \Omega, \quad t>0,
$$


with

$$
\left\{\begin{array}{l}
x=x_{1} \\
y=\sqrt{x_{2}+x_{3}^{2}+x_{4}^{2}} \\
t=t .
\end{array}\right.
$$

It is easy to check that the function $\Phi$ solves the problem in an axially symmetric domain $\Omega_{4}$ generated by a spherical rotation of $\Omega$ around of its plane of symmetry $x=0$.

Indeed, the corresponding domain $\Omega_{4}$ is then defined in $\mathbb{R}^{4}$ by:

$$
\Omega_{4}=\left\{\left(x_{1}, x_{2}, x_{3}, x_{4}\right) \in \mathbb{R}^{4} \mid x_{1}^{2}+x_{2}^{2}+x_{3}^{2}+x_{4}^{2} \leq R^{2}\right\} .
$$

We are thus led to a new problem in a five dimensional space by using $\Phi$ defined in (13). In fact, $\Phi$ satisfies the following conditions leading in particular the normal derivative constant on $\partial \Omega_{4}$.

$$
\begin{aligned}
\Delta_{4} \Phi & =\Phi_{t}, \quad \Phi>0 \text { in } \Omega_{4}, & & t>0, \\
\Phi & =0 \text { on } \partial \Omega_{4}, & & t>0, \\
\frac{\partial \Phi}{\partial \tilde{n}} & =-c(t) \text { on } \partial \Omega_{4}, & & t>0, \\
\Phi(x, 0) & =g(|x|), & & x \in \Omega_{4} .
\end{aligned}
$$

In (15), $\Delta_{4}$ denotes the Laplacian in $\mathbb{R}^{4}$ and $\tilde{n}$ appearing in (17) is an outward normal vector to the boundary $\partial \Omega_{4}$, defined by

$$
\tilde{n}=\left(\tilde{n}_{1}, \tilde{n}_{2}, \tilde{n}_{3}, \tilde{n}_{4}\right) \in \mathbb{R}^{4}, \text { with }\left\{\begin{array}{l}
\tilde{n}_{1}=n_{1}, \\
\tilde{n}_{k}=\frac{n_{2}}{y} x_{k}, k=2,3,4 .
\end{array}\right.
$$

As a second step, our aim is to prove that there exists a linear dependence between some auxiliary function $h_{i, j}$ and its normal derivative $\frac{\partial h_{i, j}}{\partial n}$, where the function $h_{, i j}$ is defined by

$$
h_{i, j}=x_{j} \Phi_{, i}-x_{i} \Phi_{, j}, \quad i, j=1,2 .
$$

We note that for simplicity sake, the subscripts in (20) will be omitted for the rest of the paper.

These functions $h$ satisfy the following conditions

$$
\begin{aligned}
\Delta h & =h_{t} \text { in } \Omega, \quad t>0, \\
\frac{\partial h}{\partial n}+K h & =0 \text { on } \partial \Omega, \quad t>0,
\end{aligned}
$$

where $K$ denotes the mean curvature of the boundary $\partial \Omega$. This last differential equality (22) results from the following lemma (see [15]). 
Lemma 2.1. Let $v(x, t) \in C^{2}(\bar{\Omega} \times(0, T))$ satisfy the boundary conditions

$$
\begin{aligned}
v(x, t) & =\Psi(t), \quad x \in \partial \Omega, \quad t \in(0, T), \\
\frac{\partial v}{\partial n} & =\gamma(t), \quad x \in \partial \Omega, \quad t \in(0, T) .
\end{aligned}
$$

Then we have

$$
\begin{aligned}
v_{n} \frac{\partial h(v)}{\partial n} & =\left(x_{i} n_{j}-x_{j} n_{i}\right) v_{n} v_{n n} \\
& =h(v) v_{n n}, \quad x \in \partial \Omega, \quad t \in(0, T) .
\end{aligned}
$$

In (24)-(25), $v_{n}$ and $v_{n n}$ stand respectively for first and second derivatives with respect to the outward normal vector $n$. Now in view of the initial condition (18), we obtain

$$
h(x, 0)=0, \quad x \in \Omega_{4} .
$$

Combining the conditions (21) and (22) together and using a classical formula of Green, we find

$$
\begin{aligned}
\int_{\Omega_{4}}|\nabla h|^{2} \mathrm{dx} & =-\int_{\Omega_{4}} h h_{t} \mathrm{dx}+\int_{\partial \Omega_{4}} h \frac{\partial h}{\partial n} \mathrm{ds} \\
& =-\frac{1}{2} \int_{\Omega_{4}}\left(h^{2}\right)_{t} \mathrm{dx}-\int_{\partial \Omega_{4}} K h^{2} \mathrm{ds},
\end{aligned}
$$

where $K$ stands for positive mean curvature of the boundary $\partial \Omega_{4}$.

Therefore we deduce that

$$
\frac{1}{2} \int_{\Omega_{4}}\left(h^{2}\right)_{t} \mathrm{dx} \leq 0
$$

Consequently, the integral of $h^{2}$ over $\Omega_{4}$ is decreasing in $t$ and in view of (26), we obtain

$$
h=0 \text { in } \Omega_{4},
$$

or

$$
\Phi=\Phi(r, t)
$$

and $\Omega_{4}$ is an $N$-ball in $\mathbb{R}^{4}$.

We conclude that the over-determined problem (1)-(3), (5) admits a solution if and only if $\Omega_{4}$ is an $N$-ball in $\mathbb{R}^{4}$, so if and only if

$$
\Omega_{4}=\left\{\left(x_{1}, x_{2}, x_{3}, x_{4}\right) \in \mathbb{R}^{4} \mid x_{1}^{2}+x_{2}^{2}+x_{3}^{2}+x_{4}^{2} \leq R^{2}=\text { const }\right\},
$$

or equivalently

$$
\Omega=\left\{(x, y) \in \mathbb{R}^{2} \mid x^{2}+y^{2} \leq R^{2}, \quad y>0\right\} .
$$

The second problem to be considered corresponds to the parameter $\alpha=2$, in this case we are concerned with the following problem 


$$
\begin{aligned}
\Delta u & =u_{t} \text { in } \Omega \times(0, \infty), \\
u(x, y, t) & =0 \text { on } \partial \Omega \times(0, \infty), \\
u(x, y, 0) & =x y g\left(\sqrt{x^{2}+y^{2}}\right), \quad(x, y) \in \Omega, \\
\frac{\partial u}{\partial n} & =-x y c(t) \text { on } \Gamma>0, \quad t>0 .
\end{aligned}
$$

With a similar reasoning as above, we set

$$
u(x, y, t)=x y v(x, y, t),
$$

and this function $v$ defined in (37) satisfies

$$
\begin{aligned}
\Delta v+2\left(\frac{v_{x}}{x}+\frac{v_{y}}{y}\right) & =v_{t}, & & v>0 \text { in } \Omega, \\
v(x, y, t) & =0 \text { on } \Gamma, & & t>0, \\
\frac{\partial v}{\partial n} & =-c(t) \text { on } \Gamma, & & t>0, \\
v(x, y, 0) & =g\left(\sqrt{x^{2}+y^{2}}\right), & & (x, y) \in \Omega .
\end{aligned}
$$

Moreover, on the two other sides of the boundary $\partial \Omega$ the normal derivative of $v$ is equal to zero. With a similar manner as above, we define $\Phi=$ $\Phi(x, y, t)$, where $x=\left(x_{1}, x_{2}, x_{3}\right), y=\left(y_{1}, y_{2}, y_{3}\right)$, by

$$
\Phi(x, y, t)=v(x, y, t), \quad(x, y) \in \Omega, \quad t>0,
$$

with

$$
\left\{\begin{array}{l}
x=\sqrt{x_{1}^{2}+x_{2}^{2}+x_{3}^{2}}, \\
y=\sqrt{y_{1}^{2}+y_{2}^{2}+y_{3}^{2}}, \\
t=t .
\end{array}\right.
$$

The corresponding domain is $\Omega_{6}$ defined in $\mathbb{R}^{6}$ by

$$
\begin{aligned}
& \Omega_{6}= \\
& \left\{\left(x_{1}, x_{2}, x_{3}, y_{1}, y_{2}, y_{3}\right) \in \mathbb{R}^{6} \mid x_{1}{ }^{2}+x_{2}{ }^{2}+x_{3}{ }^{2}+y_{1}{ }^{2}+y_{2}{ }^{2}+y_{3}{ }^{2} \leq R^{2}\right\} .
\end{aligned}
$$

In this case, the function $\Phi$ defined in (42) satisfies the following conditions (analogous to those for $\alpha=1$ ).

$$
\begin{aligned}
\Delta_{6} \Phi & =\Phi_{t}, \quad \Phi>0 \text { in } \Omega_{6}, & & t>0, \\
\Phi & =0 \text { on } \partial \Omega_{6}, & & t>0, \\
\frac{\partial \Phi}{\partial \tilde{n}} & =-c(t) \text { on } \partial \Omega_{6}, & & t>0, \\
\Phi(x, 0) & =g(|x|), & & x \in \Omega_{6},
\end{aligned}
$$


where $\Delta_{6}$ in (45) denotes the Laplacian of $\Phi$ in $\mathbb{R}^{6}$ and $\tilde{n}$ stands for the unit outward normal vector to the boundary $\partial \Omega_{6}$, defined by

$$
\tilde{n}=\left(\tilde{n}_{1}, \tilde{n}_{2}, \tilde{n}_{3}, \tilde{n}_{4}, \tilde{n}_{5}, \tilde{n}_{6}\right) \in \mathbb{R}^{6}, \text { with }\left\{\begin{array}{l}
\tilde{n}_{1}=\frac{n_{1}}{x} x_{k}, k=1,2,3, \\
\tilde{n}_{k}=\frac{n_{2}}{y} y_{k}, k=4,5,6 .
\end{array}\right.
$$

Now using the same auxiliary function $h$ defined in (20), we obtain the following transformed over-determined problem in $h$

$$
\begin{array}{rlrl}
\Delta h & =h_{t} \text { in } \Omega_{6}, & t & >, 0, \\
\frac{\partial h}{\partial n}+K h & =0 \text { on } \partial \Omega_{6}, & & t>0, \\
h(x, y, 0) & =0, \quad(x, y) \in \Omega_{6}, & t>0,
\end{array}
$$

where the condition (51) results from Lemma 2.1.

Applying the first classical formula of Green (or the monotonicity of $h^{2}$ with respect to $t$ ) or using the standard uniqueness theorem for parabolic equations [16], we claim that

$$
\Phi(x, y, t)=\Phi(r, t)
$$

and $\Omega_{6}$ is an $N$-ball in $\mathbb{R}^{6}$.

$$
\begin{array}{r}
\Omega_{6}=\left\{\left(x_{1}, x_{2}, x_{3}, y_{1}, y_{2}, y_{3}\right) \in \mathbb{R}^{6} \mid x_{1}{ }^{2}+x_{2}{ }^{2}+x_{3}{ }^{2}+y_{1}{ }^{2}+y_{2}{ }^{2}+y_{3}{ }^{2}\right. \\
\left.\leq R^{2}=\mathrm{const}\right\}
\end{array}
$$

if and only if

$$
\Omega=\left\{(x, y) \in \mathbb{R}^{2} \mid x^{2}+y^{2} \leq R^{2}, x>0, y>0 .\right\}
$$

\section{An initial PARABOlic PRoblem In $\mathbb{R}^{3}$}

This section is devoted to the following initial boundary value problem

$$
\begin{aligned}
& \Delta u=u_{t} \text { in } \Omega, \quad t>0, \\
& u(x, t)=0 \text { on } \Gamma_{0}, \quad t>0, \\
& u(x, t)=1 \text { on } \Gamma_{1}, \quad t>0, \\
& u(x, 0)=g(|x|) \text { in } \Omega \text {, } \\
& \frac{\partial u}{\partial x_{3}}=0 \text { on } \xi_{0} \cup \xi_{1}, \quad t>0 \text {, } \\
& \frac{\partial u}{\partial n}=\gamma_{i}(t) \text { on } \Gamma_{i}, \quad i=0,1, \quad t>0,
\end{aligned}
$$


where $x=\left(x_{1}, x_{2}, x_{3}\right),|x|=\sqrt{x_{1}^{2}+x_{2}^{2}+x_{3}^{2}}$ and $\Omega=\Omega_{0} \backslash \bar{\Omega}_{1}$ is a tubular region defined in $\mathbb{R}^{3}$ with

$$
\begin{array}{r}
\Omega_{i}=\left\{\left(r \cos \theta, r \sin \theta, x_{3}\right) \in \mathbb{R}^{3} \mid 0<r<r_{i}\left(\theta, x_{3}\right), 0 \leq \theta<2 \pi, 0<x_{3}<1\right\} \\
i=0,1, \quad(61)
\end{array}
$$

where $\left(r, \theta, x_{3}\right)$ are cylindrical coordinates and $0<r_{1}\left(\theta, x_{3}\right)<r_{0}\left(\theta, x_{3}\right)$ are two bounded functions of class $C^{2}, 2 \pi$ periodic in $\theta$. We assume furthermore that

$$
\frac{\partial u}{\partial x_{3}}(x, 0)=G(|x|) \quad \text { in } \Omega,
$$

where $G$ is a function of class $C^{2}$ satisfying $G=0$ on $\xi_{0} \cup \xi_{1}$.

The boundary $\partial \Omega$ is composed of two free components

$$
\begin{array}{r}
\Gamma_{i}=\left\{\left(r \cos \theta, r \sin \theta, x_{3}\right) \in \mathbb{R}^{3} \mid r=r_{i}\left(\theta, x_{3}\right), 0 \leq \theta<2 \pi, 0 \leq x_{3} \leq 1\right\}, \\
i=0,1,
\end{array}
$$

and two fixed components

$$
\begin{array}{r}
\xi_{i}=\left\{(r \cos \theta, r \sin \theta, i) \in \mathbb{R}^{3} \mid r_{1}(\theta, i) \leq r \leq r_{0}(\theta, i), 0 \leq \theta \leq 2 \pi\right\} \\
i=0,1 .
\end{array}
$$

This domain is in fact two co-axial free cylinders between the planes $x_{3}=0$ and $x_{3}=1$. Next, we consider a classical solution $u\left(x_{1}, x_{2}, x_{3}, t\right)$ of the initial parabolic problem (55)-(60), $\partial u / \partial n$ stands for the exterior normal derivative of $u$ and $\gamma_{0}(t), \gamma_{1}(t)$ are two positive functions of $t$.

In the next theorem we formulate our main result

Theorem 3.1. We assume that $\Omega_{0}$ is convex with its boundary sufficiently regular, $\Gamma_{0}$ free and

$$
\Gamma_{1}=\left\{\left(x_{1}, x_{2}, x_{3}\right) \in \mathbb{R}^{3} \mid \sqrt{x_{1}^{2}+x_{2}^{2}}=R, 0<x_{3}<1\right\} .
$$

Then the initial boundary parabolic problem (55)-(60) is solvable if and only if the components $\Gamma_{0}$ and $\Gamma_{1}$ are two co-axial circular cylinders for which the classical solution $u\left(x_{1}, x_{2}, x_{3}, t\right)$ is axially symmetric, i.e. $u(x, t)=u(r, t)$ with $r=\sqrt{x_{1}^{2}+x_{2}^{2}}$.

In order to show this statement, we split our proof in some lemmas. First of all, we investigate the auxiliary functions $h_{i, j}=x_{i} u_{, j}-x_{j} u_{, i}$ already used in the preceding sections and $W$ which we define by

$$
W:=\frac{\partial u}{\partial x_{3}} .
$$

The following lemma gives a combination between $W$ and the normal derivative of $W$ on the component $\Gamma_{0}$ which extends a result of that of [14], [17] for the elliptic case. 
Lemma 3.1. The auxiliary function defined in (65) satisfies the following differential equation on $\Gamma_{0}$

$$
\frac{\partial W}{\partial n}+2 K_{0} W=0
$$

where $K_{0}$ denotes the mean curvature of $\Gamma_{0}$.

For the proof of Lemma 3.1, we compute the normal derivative of $W$ in light of (5)

$$
\begin{aligned}
\frac{\partial W}{\partial n} & =\left(u_{, i} n_{i}\right)_{, 3}-u_{, i} n_{i, 3} \\
& =\left(u_{, i} n_{, i}\right)_{, 3}-\gamma_{0}(t) n_{i} n_{i, 3} \\
& =\left(u_{, i} n_{, i}\right)_{, 3} \\
& =n_{, i}\left(n_{i} \frac{\partial}{\partial x_{3}}-n_{3} \frac{\partial}{\partial x_{i}}\right) u_{n}+n_{3} n_{i} \frac{\partial u_{n}}{\partial x_{i}} \\
& =n_{3} \frac{\partial^{2} u}{\partial n^{2}} .
\end{aligned}
$$

Since the component $\Gamma_{0}$ is smooth enough, the differential equation (1) can be rewritten as

$$
u_{n n}+K_{0} u_{n}=u_{t}=0 .
$$

Inserting (68) into (67), we get

$$
\begin{aligned}
\frac{\partial W}{\partial n} & =-2 K_{0} n_{3} \frac{\partial u}{\partial n} \\
& =-2 K_{0} n_{3} \gamma_{0}(t) \\
& =-2 K_{0} u_{, 3}=-2 K_{0} W,
\end{aligned}
$$

and then the desired result is achieved.

The next lemma states that $u$ is independent of the third variable $x_{3}$ which leads to the conclusion that $u\left(x_{1}, x_{2}, x_{3}, t\right)=u\left(x_{1}, x_{2}, t\right)$.

Lemma 3.2. Assuming that $u$ is a classical solution of (55)-(60). Then

$$
u\left(x_{1}, x_{2}, x_{3}, t\right)=u\left(x_{1}, x_{2}, t\right) .
$$

For the proof of Lemma 3.2, we mention that the auxiliary function $W$ satisfies the following conditions

$$
\begin{aligned}
\Delta W & =W_{t} \text { in } \Omega, \quad t>0, \\
W(x, t) & =0 \text { on } \Gamma_{1} \cup \xi_{0} \cup \xi_{1}, \\
W(x, 0) & =0 \text { in } \Omega .
\end{aligned}
$$


Applying the classical formula of Green, we obtain

$$
\begin{aligned}
\int_{\Omega}|\nabla W|^{2} \mathrm{dx} & =-\int_{\Omega} W W_{t} \mathrm{dx}+\int_{\Gamma_{0}} W \frac{\partial W}{\partial n} \mathrm{ds} \\
& =-\frac{1}{2} \int_{\Omega}\left(W^{2}\right)_{t} \mathrm{dx}-2 \int_{\Gamma_{0}} K_{0} W^{2} \mathrm{ds}
\end{aligned}
$$

from which we deduce that the integral over $\Omega$ is non-positive as $\Gamma_{0}$ is convex. Hence, using (72) we conclude that $W=0$ in $\Omega$. We therefore observe that, in view of Lemma 3.2, the initial parabolic problem (55)-(60) defined in $\mathbb{R}^{3}$ can be reduced into the following 2 -dimensional one

$$
\begin{aligned}
\Delta u & =u_{t} \text { in } \omega, & & t>0, \\
u & =0 \text { on } \tilde{\gamma}_{0}=\left\{\left(x_{1}, x_{2}\right) \in \mathbb{R}^{2} \mid r=r_{0}(\theta), 0 \leq \theta<2 \pi\right\}, & & t>0, \\
u & =1 \text { on } \tilde{\gamma}_{1}=\left\{\left(x_{1}, x_{2}\right) \in \mathbb{R}^{2} \mid r=R\right\}, & & t>0, \\
\frac{\partial u}{\partial n} & =\gamma_{0}(t) \text { on } \tilde{\gamma}_{0}, & &
\end{aligned}
$$

with $r_{0}(\theta)>R$.

In (74), $\omega:=\omega_{0} \backslash \bar{\omega}_{1}$ is an annular domain where $\omega_{i}$ for $i=0,1$ is defined by

$$
\omega_{i}=\left\{\left(x_{1}, x_{2}\right) \in \mathbb{R}^{2} \mid r=\sqrt{x_{1}^{2}+x_{2}^{2}} \leq r_{i}(\theta), 0 \leq \theta<2 \pi\right\} .
$$

Lemma 3.3. We assume that $u$ is a solution of (74)-(77), where the domain $\omega$ is of class $C^{2}$ and convex and its free boundary $\tilde{\gamma}_{0}$ is of class $C^{2+\varepsilon}$. Then $\tilde{\gamma}_{0}$ is a circle centered at the origin and the solution is radial.

For the proof of Lemma 3.3, we use again the auxiliary function $h$. These functions possess the following properties

$$
\begin{aligned}
h(A u+B v) & =A h(u)+B h(v), \\
h(u v) & =u h(v)+v h(u),
\end{aligned}
$$

where $A, B$ are constants and $u, v$ are at least of class $C^{1}$.

Thus, we have:

$$
\begin{aligned}
h\left(u_{, t}\right) & =(h(u))_{, t}, \\
h\left(u_{, l} u_{, l}\right) & =2 u_{, l}(h(u))_{, l}, \\
h\left(u_{, l l}\right) & =(h(u))_{, l l} .
\end{aligned}
$$

It follows from (78)-(82) that the following conditions are satisfied

$$
\begin{aligned}
\Delta h & =h_{t} \text { in } \omega, \quad t>0, \\
h & =0 \text { on } \tilde{\gamma_{1}}, \quad t>0,
\end{aligned}
$$




$$
\frac{\partial h}{\partial n}+k_{0} h=0 \text { on } \tilde{\gamma_{0}}, \quad t>0 .
$$

This last equality results from Lemma 2.1.

In (85), $k_{0}$ stands for mean curvature of $\tilde{\gamma}_{0}$, positive. With a similar reasoning as above, we again apply the classical formula of Green and we obtain

$$
\begin{aligned}
\int_{\omega}|\nabla h|^{2} \mathrm{dx} & =-\int_{\omega} h h_{t} \mathrm{dx}+\int_{\tilde{\gamma_{0}}} h \frac{\partial h}{\partial n} \mathrm{~d} \mathrm{~s} \\
& =-\frac{1}{2} \int_{\omega}\left(h^{2}\right)_{t} \mathrm{dx}-\int_{\tilde{\gamma_{0}}} k_{0} h^{2} \mathrm{ds},
\end{aligned}
$$

from which we deduce that the integral of $h^{2}$ over $\omega$ is decreasing in $t$ and therefore in view of (58), we find $\int_{\omega}\left(h^{2}\right) \mathrm{dx} \leq 0$. Consequently, $u=u(r)$.

To this end, without using maximum principles, we can conclude that in the case of the Laplace operator the use of classical formula of Green may be considered as an alternative proof for standard uniqueness theorem for boundary value problems for both cases elliptic and parabolic, whenever the solution exists.

Acknowledgement. I am indebted to Professor Dr. Catherine Bandle from University of Basel and to Professor Antonio Greco from University of Cagliari for their comments and suggestions. I am also grateful for the comments and the thoroughly reading of the anonymas referee(s). Finally,

I would like to express my sincere thanks to Research Center of King Saud University for its support.

\section{References}

[1] Brock, F., Henrot, A., A symmetry result for an overdetermined elliptic problem using continuous rearangement abd domain derivative, Rend. Circ. Mat. Palermo (2) 51 (2002), 375-390.

[2] Dalmasso, R., Uniqueness theorems for some fourth order elliptic equations, Proc. Amer. Math. Soc. 123 (1995), 1177-1183.

[3] Dalmasso, R., Uniqueness of positive solutions for some fourth order nonlinear equations, J. Math. Anal. Appl. 201 (1996), 152-168.

[4] Dalmasso, R., Existence and uniqueness of positive solutions of semilinear elliptic systems, Nonlinear Anal. 39 (2000), 559-568.

[5] Friedman, A., Remarks on the maximum principle for parabolic equations and its applications, Pacific J. Math. 8 (1958), 201-211.

[6] Greco, A., Monotonicity of solutions to some semilinear elliptic equations, Rend. Sem. Fac. Sci. Univ. Cagliari 65(1) (1995), 17-23.

[7] Greco, A., Radial symmetry and uniqueness for an overdetermined problem, Math. Methods Appl. Sci. 24 (2001), 103-115. 
[8] Henrot, A., Philippin, G. A., On a class of over-determined eigenvalue problems, Math. Methods Appl. Sci. 20(11) (1997), 905-914.

[9] Hopf, E., A remark on elliptic differential equations of second order, Proc. Amer. Math. Soc. 3 (1952), 791-793.

[10] Hopf, E., Elementare Bemerkung über die Lösung partieller Differentialgleichungen Zweiter Ordnung von elliptischen Typus, Berliner Sitzungsber. Preuss. Akad. Wiss. 19 (1927), 147-152.

[11] Nirenberg, L., A strong maximum principle for parabolic equations, Comm. Pure Appl. Math. 6 (1953), 167-177.

[12] Payne, L. E., Isoperimetric Inequalities, Maximum Pinciples and their Applications, Lecture Notes, Univ. of Newcastle, 1972.

[13] Payne, L. E., Weinstein, A., Capacity, virtual mass, and generalized symmetrization, Pacific J. Math. 2 (1952), 633-641.

[14] Philippin, G. A., Ragoub, L., On some second order and fourth order elliptic overdetermined problems, Z. Angew. Math. Phys. 46 (1995), 188-197.

[15] Philippin, G. A., Safoui, A., Some overdetermined parabolic problems, Math. Methods Appl. Sci. 22(10) (1999), 791-799.

[16] Protter, M. H., Weinberger, H. F., Maximum Principles in Differential Equations, Sringer-Verlag, New York, 1984.

[17] Ragoub, L., Sur quelques problèmes à frontières libres de type elliptique, Thése de l'Université Laval, Québec, Canada, 1996.

[18] Serrin, J., A symmetry problem in potential theory, Arch. Rational Mech. Anal. 43 (1971), 304-318.

[19] Sperb, R., Maximum Principles and their Applications, Math. Sci. Engrg. 157, Academic Press, Inc., New York-London, 1981.

[20] Tewodros, A., Two symmetry problems in potential theory, Electron. J. Differential Equations 43 (2001), pp. 5 (electronic).

[21] Weinberger, H. F., Remark on the preceding paper of Serrin, Arch. Rational Mech. Anal. 43 (1971), 319-320.

[22] Weinstein, A., Generalized axially symmetric potential theory, Bull. Amer. Math. Soc. 59 (1952), 20-38.

L. RAgOUB

Department of Mathematics

College of Sciences

King Saud University

P.O. Box 2455, RIYADH 11451

SAUdi ARABIA

E-MAIL: RADHKLA@HOTMAIL.COM 